\title{
PADRÃO MIOELÉTRICO DO CÓLON ESQUERDO NO REFLEXO GASTROCÓLICO
}

\author{
LEFT COLON MYOELECTRIC ACTIVITY IN THE GASTROCOLIC REFLEX
}

\author{
Miguel Arcanjo dos Santos Junior, TCBC-PE ${ }^{1}$ \\ Álvaro Antônio Bandeira Ferraz, TCBC-PE ${ }^{2}$ \\ Geraldo José Paraiso Wanderley, TCBC-PE ${ }^{3}$ \\ Carlos Augusto Mathias ${ }^{4}$ \\ Fernando Antônio C. Spencer ${ }^{5}$ \\ Edmundo Machado Ferraz, TCBC-PE ${ }^{6}$
}

\begin{abstract}
RESUMO: O objetivo deste estudo foi determinar o padrão eletromiográfico do reflexo gastrocólico no cólon esquerdo (sigmóide), através da avaliação da atividade elétrica de controle (AEC), atividade elétrica de resposta (AER) e complexo motor migrante (CMM). Foram avaliadas 15 pacientes, do sexo feminino, submetidas à histerectomia, sem alteração clínica do trato gastrointestinal. A idade média foi de 40,2 anos. As pacientes foram controles de si próprias, tendo sido comparado o período pré com o per e pósprandial. Eletrodos bipolares cobertos por Téflon foram implantados a nível da tênia anterior do cólon esquerdo. Após a recuperação do íleo paralítico, realizou-se a coleta dos dados. Foi utilizado um sistema de aquisição de dados (DATA Q Série 200), que captou frequência entre 0,02 a $10 \mathrm{~Hz}$ e um sof[ware de análise de dados, (WINDAQ 200) que funcionou no ambiente Windows. Os resultados obtidos evidenciaram que a AEC e AER de curta duração (n/h) não apresentaram diferença estatística. A AER de longa duração (n/h, apresentou uma diminuição estatisticamente significativa. O CMM apresentou aumento estatístico. A conclusão do estudo foi que houve diminuição da atividade elétrica de resposta colônica de longa duração e um aumento da atividade motora do cólon esquerdc após a alimentação.
\end{abstract}

Descritores: Reflexo gastrocólico; Atividade elétrica; Motilidade.

\section{INTRODUÇÃO}

Após a alimentação, tem-se observado um aumento da atividade motora colônica ${ }^{1-7}$. Este aumento não ocorre em todas as alimentações, mas principalmente depois da primeira alimentação do dia ${ }^{8}$. Há a hipótese de que a atividade motora colônica, ou mais precisamente do retossigmóide, seja estimulada pela presença dos alimentos ${ }^{1,3,4,6,7,9,10}$. Este aumento na motilidade colônica pós-alimentar tem sido descrito como reflexo gastrocólico ${ }^{1-6,8,11-16}$.

Três fases da resposta motora colônica à alimentação têm sido descritas: a - cefálica, b - gástrica e c - intestinal ${ }^{8}$.
A fase cefálica foi descrita inicialmente, em camundongos, através da dos alimentos. A fase gástrica ocorre após a ingestão alimentar, desenvolvendo-se em torno de $30 \mathrm{mi}$ nutos, podendo persistir por até 3 horas, e a fase intestinal ocorre associada a um aumento da atividade motora no retossigmóide ${ }^{15}$. As evidências sugerem que o reflexo gastrocólico é mediado inicialmente por reflexo neurológico, vindo depois o mecanismo hormonal e finalmente a ação dos quimiorreceptores ${ }^{7-10,16-18}$.

O efeito da dieta não está estabelecido em relação às alterações eletromiográficas e motoras ${ }^{12,17}$, porém é sabia importancia da mesma na estimulação da motilidade.

1 Doutor em Cirurgia pela UFPE.

2 Professor Doutor em Bases da Técnica Cirúrgica e Anestésica pela UFPE.

3 Doutorando em Cirurgia pela UFPE.

4 Professor Doutor em Cirurgia Abdominal da UFPE.

5 Mestrando em Cirurgia pela UFPE.

6 Professor Titular e Chefe do Serviço de Cirurgia Geral HC-UFPE.

Recebido em 17/8/1999

Aceito para publicação em 5/9/2000

Trabalho realizado no laboratório de motilidade intestinal do Serviço de Cirurgia Geral do Hospital das Clínicas da Universidade Federal de Pernambuco - HC-UFPE. 
Este estudo tem como objetivo avaliar o padrão mioelétrico do cólon esquerdo no reflexo gastrocólico, pela análise da atividade elétrica de controle (AEC), atividade elétrica de resposta (AER) e complexo motor migrante (CMM).

\section{MÉ TODO}

Foram avaliadas 15 pacientes, todas do sexo feminino, provenientes do Serviço de Ginecologia da UFPE, com indicação de histerectomia por mioma uterino, associado a sangramento vaginal. A faixa etária variou de 33 a 51 anos, com média de 40,2 anos e desvio padrão de 5,5 anos. Foram excluídas do grupo de estudo pacientes com idade acima de 55 anos e abaixo de 30 anos, pacientes diabéticas e constipadas cronicas, tendo sido utilizado como método de triagem a história clínica e a medição da glicemia de jejum.

O protocolo de estudo foi submetido à avaliação da Comissão de Ética do Conselho Regional de Medicina, tendo sido obtida a aprovação e recebida autorização para a sua realização.

As pacientes selecionadas para o procedimento receberam explanação verbal, realizada pelo médico assistente, e escrita, do presente protocolo. Apenas as pacientes que concordaram com os propósitos do estudo foram selecionadas para a aposição dos eletrodos.

A técnica cirúrgica empregada foi histerectomia total, com incisão Pfannenstiel, com tempo cirúrgico médio de 125,3 minutos, no serviço de Cirurgia Geral (SCG) do Hospital das Clinicas da UFPE.

As 15 pacientes, após a histerectomia total e completa recuperação do íleo paralítico, representada pela eliminação de fezes e flatos, foram levadas ao laboratório de motilidade do Serviço de Cirurgia Geral do Hospital das Clínicas da UFPE, analisando-se os dados antes, durante e depois da alimentação padrão.

Ao término do procedimento cirúrgico, caso não houvesse qualquer intercorrência durante o mesmo, eletrodos bipolares, cobertos por Teflon (fio de marca-passo - monicrom 2 - 0 - aço cirúrgico revestido para marca-passo de $60 \mathrm{~cm}$ ) eram implantados ao nível da tênia anterior do cólon, na camada muscular. Foram implantados três pares de eletrodos no cólon esquerdo, cada um distando $5 \mathrm{~cm}$ do outro. $\mathrm{O}$ último dos eletrodos do cólon esquerdo distava $20 \mathrm{~cm}$ da reflexão peritoneal. Os eletrodos foram expostos por uma agulha, presos a uma das extremidades no flanco esquerdo e fixados à pele, tendo sido gasto uma média de 9 min na aposição dos eletrodos.

Após a recuperação do íleo paralítico, com a presença de eliminação de fezes e flatos, e o paciente em dieta livre, mas com jejum de 12 horas antes da realização da leitura e recebendo uma dieta padrão de 823,4 cal, (orientada pelo serviço de nutrição do Hospital das Clínicas da UFPE), foi realizada a coleta dos dados. A dieta foi constituída de: hidratos de carbono - 448,36 cal (112,09g, 54\%); proteínas - 46,64 cal $(11,66 \mathrm{~g}, 6 \%)$; e gordura $-328,05 \mathrm{cal}$ $(36,45 \mathrm{~g}, 40 \%)$. Esta dieta foi de sanduíche com pão branco $(46 \mathrm{~g})$, carne (132g), maionese (22g), suco de laranja
$(150 \mathrm{~g})$; foi realizada a leitura da atividade mioelétrica colônica, que ocorreu em média no quarto dia de pós-operatório. Os períodos de leitura foram o pré-prandial, per-prandial e pós-prandial. O tempo médio de leitura foi de 57 minutos para o pré-prandial, 11,3 minutos para o per-prandial e de 63 minutos para o pósprandial.

Os sinais foram enviados a um computador, através de um sistema de aquisição de dados, que capta freqüência entre 0,02 e $10 \mathrm{~Hz}$. Antes dos dados serem registrados na placa, eram amplificados com um ganho de até 1000 vezes, e armazenados. Utilizou-se um programa de análise de dados. A aquisição de dados se deu com uma mostragem de $40 \mathrm{~Hz}$ por segundo. Como o sinal do intestino apresenta uma amplitude muito pequena, sendo acompanhada por um ruído, de modo comum, de amplitude muitas vezes superior ao próprio sinal, foi idealizado um circuito contendo como estágio de entrada um amplificador de instrumentação especial, permitindo relação de ganho de 1 a 1000, uma excelente razão de rejeição e uma altíssima impedancia de entrada. Neste estágio, grande parte do ruído de modo comum é eliminado, desde que se assegure um equilíbrio de impedancias conectadas a cada uma das portas inversoras e não inversoras do amplificador. Em seguida, tem-se um filtro de Butterworth de quarta ordem, com frequiência de corte em torno de $15 \mathrm{~Hz}$, atenuando ainda mais o ruído de modo comum, que apresenta uma frequência média de $60 \mathrm{~Hz}$. Por fim, aplica-se um novo ganho ao sinal, tornando-o compatível com as características de entrada do sistema.

O programa de análise dos dados realiza uma análise através de algorritmos baseados na Fast Fourier Transform (FFT). O programa usa o FFT para depurar e analisar os dados. O programa FFT tem uma resolução de 0,23 ciclos/ minuto. Feito isso, ocorre a separação da AEC e da AER. As frequências situadas abaixo de $0,3 \mathrm{~Hz}$ são selecionadas para a AEC, enquanto que, os dados situados entre 0,7 a 10 $\mathrm{Hz}$, para a análise da AER.

$\mathrm{Na}$ análise da AEC, determinam-se as freqüências dominantes e suas respectivas percentagens, além da realização da disposição gráfica dos dados. A frequiência dominante é aquela que apresenta a maior magnitude. A freqüência dominante, durante cada minuto, foi classificada de acordo com o número de ciclos por minuto em: baixa (0-9 ciclos/minuto), média (10-15 ciclos/minuto) e alta (acima de 15 ciclos/minuto).

O sinal da AER deve ter duração mínima acima de 0,6 s e uma média de amplitude maior que da linha basal. O inicio e o fim do tempo e duração de cada atividade elétrica de resposta foram classificados como de curta $(<6,7 \mathrm{~s})$ ou longa $(>6,7 \mathrm{~s})$ duração.

Para rejeitar artefatos ou ruídos, a atividade elétrica de resposta, que começa simultaneamente e que tem a mesma duração em todos os eletrodos, no mesmo segmento colônico, é rejeitada e considerada como interferência.

O CMM foi identificado através da análise visual da AER de longa duração, que ocorreu nos 3 eletrodos, com um intervalo de 0,2 segundos entre uma e outra atividade elétrica, tanto no sentido proximal como distal. 
Realizou-se ainda uma análise espectral e morfológica dos traçados elétricos, para afastar possíveis deslocamentos dos eletrodos da camada muscular do cólon esquerdo.

$\mathrm{Na}$ análise estatística dos dados, considerando-se, inicialmente, de acordo com o teorema do limite central, que as amostras se distribuíam de maneira normal, foi utilizado o teste " $t$ " de Student bicaudal para amostras relacionadas. Ainda foi realizado teste de aderência, para demonstrar a distribuição normal da amostra.

Um valor de $\mathrm{p}<0.05$ foi considerado como indicativo de diferença estatisticamente significativa.

\section{RESULTADOS}

Os pacientes submetidos a cirurgia não tiveram complicações pós-operatória, e também não houve morbidade relacionada à aposição dos eletrodos (retirados, por tração, em média no quinto dia de pós-operatório).

O eletrodo um do paciente três, estava fora da sua posição, identificado através da análise gráfica visual do traçado, e foi excluído do estudo.

Na Tabela 1 foi exposta a AEC, comparando o período pré-prandial com o prandial e pós-prandial. Ob- servou-se que não houve diferença estatisticamente significativa.

Na Tabela 2, foi exposta a AER de curta duração (número/hora), não tendo ocorrido diferença estatisticamente significativa, comparando o período pré-prandialcom o per e pós-prandial.

Na Tabela 3 foi exposta a AER de curta duração, em relação a duração, tendo sido evidenciada uma diminuição estatisticamente significativa, quando comparado o período pré-prandial com o pós-prandial, de zero e quinze e zero a trinta minutos.

Na Tabela 4 foi exposta a AER de longa duração, em relação ao número por hora, e foi evidenciada uma diferença estatisticamente significativa, quando comparado o período pré-prandial com o prandial e o pós-prandial, nos intervalos de zero a quinze, zero a quarenta e cinco e zero a sessenta minutos.

Na Tabela 5 foi exposta a duração da AER de longa duração, onde observou-se diferença estatisticamente significativa, quando comparados os períods pré-prandial com o pós-prandial.

Na avaliação do CMM, Tabela 6, observou-se diferença estatisticamente significativa entre o período préprandial com o prandial.

Tabela 1

Discriminação dos resultados da atividade elétrica de controle nos grupos estudados (\%)

\begin{tabular}{|c|c|c|c|c|}
\hline Período & $n$ & $A E C$ & Desvio padrão & $p^{*}$ \\
\hline Controle & 15 & 98,02 & 1,84 & \\
\hline Prandial & 15 & 98,31 & 2,43 & 0,72 \\
\hline $\begin{array}{c}\text { Pós-prandial } \\
0-15 \text { min }\end{array}$ & 15 & 97,07 & 2,86 & 0,14 \\
\hline $0-30 \mathrm{~min}$ & 15 & 97,2 & 2,9 & 0,31 \\
\hline $0-45 \min$ & 10 & 96,9 & 2,42 & 0,13 \\
\hline $0-60 \mathrm{~min}$ & 8 & 97,3 & 2,8 & 0,47 \\
\hline
\end{tabular}

*p relacionado ao período pré-prandial (controle).

Tabela 2

Discriminação dos resultados da atividade elétrica de resposta de curta duração (número / hora)

\begin{tabular}{|c|c|c|c|c|}
\hline Período & $n$ & $A E R$ & Desvio padrão & $p^{*}$ \\
\hline Controle & 15 & 98,6 & 27,3 & \\
\hline Prandial & 15 & 97,8 & 23,2 & 0,88 \\
\hline $\begin{array}{c}\text { Pós-prandial } \\
0-15 \text { min }\end{array}$ & 15 & 107,2 & 19,7 & 0,12 \\
\hline $0-30 \mathrm{~min}$ & 15 & 101,6 & 16,9 & 0,54 \\
\hline $0-45 \mathrm{~min}$ & 10 & 99,7 & 17,7 & 0,29 \\
\hline $0-60 \mathrm{~min}$ & 8 & 101,3 & 22,5 & 0,40 \\
\hline
\end{tabular}

*p relacionado ao período pré-prandial (controle). 
Tabela 3

Discriminação dos resultados da atividade elétrica de resposta de curta duração (Duração - segundos)

\begin{tabular}{lcc|c|c}
\hline Período & $n$ & AER (duração $-s)$ & Desvio padrão & $p^{*}$ \\
\hline Controle & 15 & 2,3 & 0,3 & 0,057 \\
Prandial & 15 & 2,1 & 0,3 & 0,002 \\
Pós-prandial & 15 & & & 0,02 \\
$0-15$ min & 15 & 2,1 & 0,27 & 0,09 \\
$0-30$ min & 10 & 2,9 & 0,29 & 0,45 \\
$0-45$ min & 8 & 2,3 & 1,33 & \\
$0-60 \mathrm{~min}$ & & 2,74 & & \\
\hline
\end{tabular}

*p relacionado ao período pré-prandial (controle).

Tabela 4

Discriminação dos resultados da atividade elétrica de resposta de longa duração (número/hora).

\begin{tabular}{lcccc}
\hline $\begin{array}{c}\text { Período } \\
\text { (número/hora) }\end{array}$ & $n$ & AER (Longa duração) & Desvio padrão & $p^{*}$ \\
\hline Controle & 15 & 8,1 & 3,2 & 0,04 \\
Prandial & 15 & 5,7 & 3,5 & 0,032 \\
Pós-prandial & & & 4,3 & 0,054 \\
$0-15$ min & 15 & 6,2 & 2,77 & 0,0008 \\
$0-30$ min & 15 & 6,31 & 2,13 & 0,02 \\
$0-45$ min & 10 & 5,46 & 3,0 & 0 \\
$0-60$ min & 8 & 6,9 & & \\
\hline
\end{tabular}

*p relacionado ao período pré-prandial (controle).

Tabela 5

Discriminação dos resultados da atividade elétrica de resposta de longa duração (Duração - segundos)

\begin{tabular}{lcccc}
\hline Período & $\mathrm{n}$ & AER (duração-s) & Desvio padrão & $\mathrm{p}^{*}$ \\
\hline Controle & 15 & 11,3 & 0,35 & 0,08 \\
Prandial & 15 & 9,97 & 0,31 & 0,00022 \\
Pós-prandial & & & & 0,0048 \\
0-15 min & 15 & 7,03 & 2,97 & 0,009 \\
$0-30$ min & 15 & 8,7 & 3,43 & 0,002 \\
$0-45$ min & 10 & 9,42 & 3,04 & 2,83 \\
$0-60$ min & 8 & 8,71 & & \\
\hline
\end{tabular}

*p relacionado ao período pré-prandial (controle).

Tabela 6

Discriminação dos resultados do Complexo Motor Migrante (CMM)

\begin{tabular}{lcccc}
\hline Período & $n$ & CMM & Desvio padrão & $p^{*}$ \\
\hline Controle & 15 & 0,8 & 0,67 & 0,0036 \\
Prandial & 15 & 6,4 & 6,19 & 0,42 \\
Pós-prandial & 15 & 1,00 & 0,75 & 0,75 \\
\hline
\end{tabular}

*p relacionado ao período pré-prandial (controle). 


\section{DISCUSSÃO}

A análise através de um programa de computador teve a vantagem de ser objetiva e uma grande quantidade de dados pôde ser analisada, sem erros ou tendenciosidade, os quais poderiam ocorrer com a análise gráfica visual ${ }^{19-23}$.

A aposição de eletrodos na serosa necessitou de uma laparotomia; esse método, além de invasivo, pode ter acarretado alterações da leitura, pois houve o estresse cirúrgico, assim como a utilização de drogas anestésicas e analgésicos no pós-operatório ${ }^{19-23}$.

Como tivemos de realizar uma laparotomia para a aposição dos eletrodos, pode-se interrogar quais as repercussões da mesma sobre a motilidade e atividade elétrica colônica. Graber et $\mathrm{al}^{26}$, Condon et $\mathrm{al}^{27}$ estudaram a recuperação do íleo paralítico e não foi evidenciada relação do porte cirúrgico com o tempo de resolução do íleo. Waldhausen et al $^{16}$ estudaram o efeito da deambulação precoce na resolução do íleo paralítico, utilizando fios de marcapasso na camada seromuscular, e não encontraram dados que corroborassem esta proposição. Apesar de ter sido um método invasivo e, também associado com o trauma cirúrgico, os trabalhos que versaram sobre este assunto não demonstraram existir alteração na interpretação da leitura elétrica e mecanica, depois da recuperação do íleo paralítico ${ }^{19-24}$.

Para se conseguir uma leitura adequada da atividade elétrica, utilizamos a análise matemática de Fourier (Fast Fourier transform - FFT) ${ }^{22}$, usada para determinar os componentes de frequência da atividade colônica de onda lenta Essa foi uma técnica utilizada para descrever uma onda não sinusoidal como uma série de ondas sinusoidais, nas quais as frequências foram relatadas para cada uma delas como integrais de baixa frequência. Através da utilização da análise de Fourier, foi possivel avaliar as ondas do cólon, que foram lentas, de frequência irregular, em contraste com aquelas do estômago e do intestino delgado.

A alimentação tem sido associada com o aumento da peristalse intestinal ${ }^{1-3,6,8-12,17,18,27,28}$. As gorduras apresentaram uma maior resposta motora colônica, quando comparadas com a estimulação por aminoácidos e / ou carboidratos $4,6,9,10,28$; houve até diminuição da peristalse quando da aplicação de dieta à base de aminoácidos ${ }^{4}$. Então, como estávamos estudando um reflexo fisiológico, foi pór nós utilizada uma dieta padrão estabelecida para a nossa população.

Dentro do estudo da ação dos alimentos no reflexo gastrocólico, Daly et al ${ }^{10}$ estudaram o efeito da comida e drogas anticolinérgicas, nas contrações próprias do retossigmóide em voluntários. Em relação à alimentação, observaram que, durante o período pré-prandial, a motilidade retossigmoideana consistiu em contrações isoladas. Após a alimentação, houve aumento significativo na ocorrência de contrações simultaneas, com- pequena redução nas contrações do canal de leitura. Em nosso estudo, no período pré-prandial, todos os pacientes apresentavam atividade elétrica e motora e, observamos diminuição da AER significativa, assim como aumento nas contrações migratórias durante a alimentação, o que foi compatível com o aumento da motilidade colônica.
Na avaliação da atividade motora colônica, observou-se aumento significativo das contrações migratórias no período prandial, o que provavelmente está associado à alteração neurormonal e, no período pós-prandial, não houve diferença quando comparado este ao período de controle. A chegada do alimento ao estômago determinou aumento da motilidade colônica esquerda.

A motilidade colônica foi demonstrada no nosso estudo através da atividade elétrica de controle, atividade elétrica de resposta e complexo motor migrante.

Alguns trabalhos avaliaram a pressão intraluminal diferentemente do nosso. Kelin et $\mathrm{al}^{3}$, medindo a pressão intraluminal e o índice de motilidade no íleo, ascendente, retossigmóide e reto, comparando o período de jejum com o pós-prandial, observaram que não houve relação entre a atividade motora do ascendente e a do cólon distal; o índice de motilidade foi modificado pela dieta balanceada, mas não houve alteração quando a dieta foi feita com aminoácidos. Esta metodologia utilizou eletrodos intraluminares, o que necessitou de limpeza colônica, também avaliou pressão, diferentemente do nosso estudo, que avaliou a atividade elétrica e motora, com eletrodos de seromuscular, os quais apresentaram resultados mais precisos ${ }^{20}$. Na nossa avaliação, observou-se um aumento da atividade motora (CMM), estatisticamente significativo, quando comparado o período pré-prandial com o per-prandial. Em relação ao período pós-prandial houve aumento, porém não estatisticamente significativo.

$\mathrm{Na}$ avaliação da atividade elétrica, Dapoigny et al ${ }^{11}$ estudaram comparativamente a atividade mioelétrica do cólon direito, esquerdo e retossigmóide, através da atividade elétrica de controle e da atividade elétrica de resposta e, observaram que havia diferença em relação à duração ou à amplitude nos dois tipos de onda, nos três segmentos colônicos estudados, tanto em relação ao período pré-prandial e pós-prandial, como em relação ao de repouso. Antes da alimentação, o número da atividade elétrica de resposta foi menor no cólon direito do que no esquerdo e no retossigmóide. Após a alimentação, houve aumento significativo na atividade elétrica de resposta nos primeiros $20 \mathrm{mi}$ nutos no cólon direito e, em 100 minutos no cólon esquerdo e no retossigmóide. A atividade elétrica de controle não apresentou diferença durante o período de observação. Estes resultados evidenciaram a heterogeneidade nas diferentes partes colonicas, com relativa hipomotilidade no cólon direito, comparado ao esquerdo e ao retossigmóide, em seres humanos sadios ${ }^{4}$. 0 que nós observamos no cólon esquerdo, quando comparado o período pré-prandial com o pósprandial, foi que a AEC também não apresentou diferença estatística, assim como a AER de curta duração em relação ao número por hora, e que a AER de longa duração apresentou uma diminuição estatisticamente significativa, diferentemente do que acharam Dapoigny et $\mathrm{al}^{11}$. Como a atividade elétrica de resposta foi responsável por contrações, de pequena ou longa duração, a diminuição do número por hora se deu provavelmente pelo aumento na sua duração, o que determinou aumento dos complexos motores migrantes, os quais ocorreram tanto em sentido distal como proximal, determinando a movimentação do conteú- 
do fecal, assim como absorção de água e eletrólitos. Se houvesse um aumento em sentido distal único, provavelmente teríamos evacuações com frequência após cada alimentação.

No nosso estudo, realizado em adultos, houve aumento no complexo motor migrante, estatisticamente significativo quando comparado o periodo pré com o per-prandial e, não foi observado necessidade de evacuação no periodo pós-prandial. E ainda, as contrações colonicas apresentaram um percentual de $46 \%$ e $65 \%$ no sentido proximal, no periodo pré-prandial e pós-prandial, respectivamente, o que corroborou a função de mistura e absorção colonica. No periodo per-prandial, existe uma possível ação neurohormonal, pois mesmo não havendo tempo para chegar o alimento ao cólon, houve aumento do complexo motor migrante.

Apesar de termos realizado o estudo em humanos, observamos aumento do complexo motor migrante, quando comparamos os periodos pré, per e pós-prandiais; porém, não foi estatisticamente significativo em relação ao pós-prandial. E mais, todos os pacientes apresentavam atividade elétrica no período pré-prandial e, portanto, não estavam no período quiescente, o que foi compatível com a diminuição da atividade elétrica de controle.

A nossa avaliação do período pós-prandial resultou na média de 63 minutos, não tendo sido estudada a resposta tardia. Observamos nos primeiros 15 minutos, diminuição da AER de curta duração (número/hora) não estatisticamente significativa e diminuição significativa da AER de longa duração (número/hora). A duração da AER, tanto de curta, como de longa duração, apresentou diminuição estatisticamente significativa. Porém, houve aumento não significativo do complexo motor migrante, o que demonstrou um aumento na motilidade colônica no período pósprandial, compatível com as funções de eliminação colônica pós-alimentar.

Em nosso estudo, observamos um aumento significativo da motilidade colônica durante a alimentação e um aumento não significativo no período pós-prandial, o que se deu, provavelmente, pela presença do alimento no estômago, que através de estímulos neurormonal. A presença contínua de fezes na luz intestinal talvez tenha sido o fator responsável pelo aumento não significativo da contratilidade no reflexo gastrocólico, no período pós-prandial, pois quando comparado em indivíduos com preparo colônico, houve aumento nos mesmos.
$\mathrm{Na}$ nossa avaliação, todos os pacientes estavam com o transito intestinal normal, com eliminação de fezes e flatos, e a colocação dos eletrodos se deu durante a laparotomia. Portanto, a leitura foi feita o mais próximo possível do estado fisiológico, e foi evidenciado um aumento significativo da atividade motora no período prandial e uma diminuição significativa da AER de longa duração. Em nenhum dos 15 pacientes foi identificado um período de ausência de atividade elétrica colônica no período pré-prandial, o que, provavelmente, foi responsável pela ausência de aumento significativo na atividade elétrica.

A remoção do conteúdo fecal pela lavagem, assim como a mudança da dieta para o preparo colônico, foram fatores que alteraram a fisiologia da motilidade colônica, podendo ter alterado a interpretação eletromiográfica. Também, a utilização de eletrodos na camada mucosa e não na seromuscular, pode ter sido responsável pelas alterações eletromiográficas.

Nossos achados sugerem um aumento na atividade motora colônica, no período pósprandial, a nível de retossigmóide. Kerlin et $\mathrm{al}^{3}$ também descreveram aumento da atividade motora no período pós-prandial. O mecanismo do aumento da motilidade colônica pós-prandial, não foi esclarecido, parecendo ser de origem neurormonal. Apesar dos hormônios sofrerem um aumento nos primeiros 30 minutos após a alimentação, a sua concentração plasmática permanece elevada por horas. Como o aumento ocorreu a nível de retossigmóide, apesar de níveis hormonais elevados (sérico), deve haver um número maior ou uma maior sensibilidade a estes hormônios do que no restante do cólon.

A conclusão, após avaliação da atividade elétrica e motora do cólon sigmóide, foi que não houve aumento significativo da atividade elétrica de controle e de resposta de curta duração; houve uma diminuição da atividade elétrica de resposta de longa duração e um aumento significativo nas contrações migratórias no período prandial, havendo portanto uma diminuição de contratilidade e um aumento de propagação colônica (sigmóide). Uma extensão de nossa pesquisa seria a avaliação do cólon direito e transverso, onde poderíamos encontrar resultados diferentes daqueles evidênciados no cólon esquerdo.

\begin{abstract}
Our aim in this study was to settle the patterns of the myoelectric activity on the left colon ( sigmoid) regarding the gastrocolic reflex by analysing the Electric Control Activity (ECA), the Electric Response Activity (ERA), the Migrating Colonic Contractions. We included 15 patients, with no gastrointestinal tract complaints, who underwent histerectomy. The mean age was 40,2 years. Patients were control of themselves and registers were compared with each other before, during and after meal. Bypolar electrodes covered with teflon were implanted on the anterior left colon taenia. After postoperative recovery, data collection was put together. There was taken a DATA $Q 200$ system for the collection data which reaches frequencies between 0,02 and $10 \mathrm{~Hz}$ and a software that runs in the Windows environment. Our results showed that there was no significant difference neither in the ECA nor
\end{abstract}


in the short duration ERA ( number/hour) between the groups. The long duration ERA showed a significant decrease regarding its number/hour. There was a statistically significant difference in the Migrating Motor Complex between the registers before and during meal. Regarding the left colon, we concluded that while the electric activity decreased, the motor activity increased after meal.

Key words: Gastrocolic response; Electric activity; Motility.

\section{REFERÊNCIAS}

1. Bassotti G, Morelli A, Whitehead W - Abnormal rectosigmoid myoelectric response to eating in patients with severe idiopathic constipation. Disease Colon Rectum, 1992, 35:753-756.

2. Bassotti G, Betti C, Imbimbo BP et al. - Colonic Motor Response to Eating: A Manometric Investigation in Proximal and Distal Portions of the Viscus in Man. American Journal of Gastroenterology, 1989, 84(2): 118-122.

3. Kerlin P, Zinsmeister A, Phillips S. Motor responses to food of the ileum, proximal colon, and distal colon of healthy humans. Gastroenterology, 1983, 84:762-770.

4. Levinson S, Bhasker M, Gibson TR, et al - Comparison of intraluminal and intravenous mediators of colonic response to eating. Digestive Diseases and Sciences, 1985, 30(1): 33-39..

5. Schulte WJ, Cowles VE, Condon RE - Hypokalemia and the gastrocolic response. Digestive Diseasesand Sciences 1984,29:A551.

6. Sims MA, Hasler WL, Chey WD et al - Hyperglycemia inhibits mechanoreceptormediated gastrocolonic responses and colonic peristaltic reflexes in healthy humans. Gastroenterologv, 1995, 108: 350-359.

7. Wiley J, Tatum D, Keinath R. Participation of gastric mechanoreceptors and intestinal chemoreceptors in the gastrocolonic response. Gastroenterology, 1988, 94: 1144-1149.

8. Sarna SK, Lang IM - Colinic Motor response to a meal in dogs American Joúnal of Physiology. 1989, 257(2): G830-G835.

9. Battle WM, Cohen S, Snape WJJR - Inhibition of postprandial colonic motility after ingestion of an amino acid mixture. Digestive Diseases and Sciences, 1980, 25(9): 647-652.

10. Daly J, Bergin A, Sun WM et al - Effect of food and anticholinergic drugs on the pattern of rectosigmoid contractions. Gut, 1993, 34: 799-802.

11. Dapoigny M, Trolese JF, Bommelaer G, et al - Myoelectric spiking activity of right colon, left colon, and rectosigmoid of healthy humans. Digestive Disease and Sciences, 1988, 33(8): 1007-1012.

12. Lémann M, Flourié B, Picon L, et al - Is the motor activity different in the unprepared and prepared human colon? Motility and Nerve-gut Interactions, 1991, A463.

13. Medeiros JAS, Pontes FA, Mesquita OARO - Is colonic electrical activity a similar phenomena to small-bowel electrical activity? Disease of the Colon and Rectum, 1997, 40(1): 93-99.

14. O'Brien MD, Camilleri M, Von Der Ohe MR, et al - Motility and tone of the left colon in constipation: a role in clinical practice? American Journal of Gastroenterology, 1996, 91(12): 2532-2538.
15. Snape Jr WJ, Wright SH, Battle WM et al - The gastrocolic response: Evidence for a neural mechanism. Gastroenterology. 1979, 77: 1235-1240.

16. Waldhausen JHT, Schirmer BD - The effect of ambulation on recovery from postoperative ileus. Annals of Surgery, 1990, 212(6): 671-677.

17. Sarna SK - Cyclic motor activity: migration motor complex. Gastroenterology, 1985, 89: 894-913.

18. Snape JR WJ, Matarazzo AS, Cohen S - Effect of eating and gastrointestinal hormones in human colonic myoecectrical and motor activity. Gastroenterology. 1978, 75: 373-378.

19. Condon RE, Cowles VE, Ferraz AAB et al - Human colonic smooth muscle electrical activity during and after recovery from postoperative ileus. American Journal of Physiology, 1995, G408-G417.

20. Ferraz AAB, Bacelm TS, Santos JR MA, et al - Avaliação mioelétrica do cólon: descrição de metodologia. Revista Brasileira de Colo-Proctologia, 1995, 15(3): 114-121.

21. Santos JR, MA, Ferraz AAB, Wanderley GJP, et al - Padrão mioelétrico do cólon esquerdo em pacientes portadores de esquistossomose mansônica na forma hepatoesplênica. Anais da Faculdade de Medicina da Universidade Federal de Pernambuco, Recife, 1996, 41 (1): 3-10.

22. Sunshine AG, Perry R, Reynolds JC et al - Colonic slowwave analysis. Limitations of usefulness of fast Fourier transform (FFT). Digestive Diseases and Sciences, 1989, 34(8): 1173-1179.

23. Vigmond EJ, Bardakjian BL - The effect of morphological interdigitation on field coupling between smooth muscle cells. IEEE Transactions On-Biomedial Engineering" 1995, 42(2): 162-171.

24. Cowles VE, Condon RE, Ferraz AAB et al - Computer analysis of human colonic smooth muscle electrical activity. Gastroenterology. In prelo.

25. Sarna SK, Waterfall WE, Bardakjian BL - Types of human colonic electrical activities recorded postoperatively. Gastroenterology, 1981, 81: 61-70.

26. Graber JN, Schulte WJ, Condon RE et al. Relationship of duration of postoperative ileus to extent and site of operative dissection. Surgery, 1982, 92(1): 87-92.

27. Condon RE, Verne FACS, Cowles BS et al. The effect of whole-gut lavage on colon motility and gastrocolonic response in a monkey. Surgery, 1986, 531536.

28. Wright SH, Snape JR WJ, Battle WM et al. Effect of dietary components on the gastrocolonic response. American Journal of Phvsiology, 1980, 238: G228232.

Endereço para correspondência:

Dr. Miguel Arcanjo Santos Júnior

Rua 48, 165/304

52020-060 - Recife-PE 